\title{
Two alternative recessive quantitative trait loci influence resistance to spring black stem and leaf spot in Medicago truncatula Lars G Kamphuis ${ }^{1}$, Judith Lichtenzveig ${ }^{1,2}$, Richard P Oliver ${ }^{1}$ and Simon R Ellwood*1
}

Address: ${ }^{1}$ Australian Centre for Necrotrophic Fungal Pathogens, State Agricultural Biotechnology Centre, Murdoch University, Perth 6150 , Western Australia, Australia and ${ }^{2}$ Commonwealth Scientific and Industrial Research Organisation, Plant Industry, Private Bag No. 5, Wembley 6913, Western Australia, Australia

Email: Lars G Kamphuis - Lars.Kamphuis@csiro.au; Judith Lichtenzveig - j.lichtenzveig@murdoch.edu.au; Richard P Oliver - R.Oliver@murdoch.edu.au; Simon R Ellwood*-S.Ellwood@murdoch.edu.au

* Corresponding author

Published: 26 March 2008

BMC Plant Biology 2008, 8:30 doi:10.1 I86/147|-2229-8-30
Received: II December 2007

Accepted: 26 March 2008

This article is available from: http://www.biomedcentral.com/I47I-2229/8/30

(c) 2008 Kamphuis et al; licensee BioMed Central Ltd.

This is an Open Access article distributed under the terms of the Creative Commons Attribution License (http://creativecommons.org/licenses/by/2.0), which permits unrestricted use, distribution, and reproduction in any medium, provided the original work is properly cited.

\begin{abstract}
Background: Knowledge of the genetic basis of plant resistance to necrotrophic pathogens is incomplete and has been characterised in relatively few pathosystems. In this study, the cytology and genetics of resistance to spring black stem and leaf spot caused by Phoma medicaginis, an economically important necrotrophic pathogen of Medicago spp., was examined in the model legume $M$. truncatula.
\end{abstract}

Results: Macroscopically, the resistant response of accession SA27063 was characterised by small, hypersensitive-like spots following inoculation while the susceptible interaction with accessions AI7 and SA3054 showed necrotic lesions and spreading chlorosis. No unique cytological differences were observed during early infection $(<48 \mathrm{~h})$ between the resistant and susceptible genotypes, except pathogen growth was restricted to one or a few host cells in SA27063. In both interactions reactive oxygen intermediates and phenolic compounds were produced, and cell death occurred. Two $F_{2}$ populations segregating for resistance to spring black stem and leaf spot were established between SA27063 and the two susceptible accessions, AI7 and SA3054. The cross between SA27063 and AI 7 represented a wider cross than between SA27063 and SA3054, as evidenced by higher genetic polymorphism, reduced fertility and aberrant phenotypes of $F_{2}$ progeny. In the SA27063 $\times$ AI7 $F_{2}$ population a highly significant quantitative trait locus $(\mathrm{QTL}, \mathrm{LOD}=7.37 ; P<0.0000 \mathrm{I})$ named resistance to the necrotroph Phoma medicaginis one $(\mathrm{rnpm} /)$ genetically mapped to the top arm of linkage group 4 (LG4). rnpml explained $33.6 \%$ of the phenotypic variance in the population's response to infection depicted on a I-5 scale and was tightly linked to marker AW256637. A second highly significant QTL (LOD = 6.77; P < 0.0000 I), rnpm2, was located on the lower arm of LG8 in the SA27063 × SA3054 map. rnpm2 explained $29.6 \%$ of the phenotypic variance and was fine mapped to a $0.8 \mathrm{cM}$ interval between markers $\mathrm{h} 2$ _ $16 \mathrm{a} 6 \mathrm{a}$ and h2_2 IhIld. rnpm I is tightly linked to a cluster of Toll/Interleukin I receptor-nucleotide binding site-leucine-rich repeat (TIRNBS-LRR) genes and disease resistance protein-like genes, while no resistance gene analogues (RGAs) are apparent in the genomic sequence of the reference accession AI7 at the rnpm2 locus.

Conclusion: The induction of defence responses and cell death in the susceptible interaction following infection by $P$. medicaginis suggested this pathogen is not negatively affected by these responses and may promote them. A QTL for resistance was revealed in each of two populations derived from crosses between a resistant accession and two different susceptible accessions. Both loci are recessive in nature, and the simplest explanation for the existence of two separate QTLs is the occurrence of host genotype-specific susceptibility loci that may interact with undetermined $P$. medicaginis virulence factors. 


\section{Background}

Phoma medicaginis is the causal agent of spring black stem and leaf spot in alfalfa (Medicago sativa), a major fodder and forage crop in temperate and Mediterranean regions. Studies in four north-eastern US states have shown foliar necrotrophs, principally $P$. medicaginis, resulted in estimated average yield losses of over 13\%. For harvests where significant yield losses occurred, this figure rose to over $19 \%$ [1].

P. medicaginis also causes disease on the model legume Medicago truncatula, or barrel medic, which is used in Australia in ley rotations to enhance soil nitrogen [2]. In susceptible cultivars, reduction in seed and herbage yields, and almost complete defoliation and premature death has been reported $[3,4]$, with infected cultivars exhibiting an average seed weight reduction of 37.3\% [5]. Chemical and cultural control of foliar necrotrophs like $P$. medicaginis has proved to be expensive and inefficient [1], and therefore new resistant cultivars or more efficient antifungal control agents are required.

Since the early 1990s, resistance genes (R genes) against various pathogens and pests have been isolated from important crop species such as barley, tomato and rice [reviewed in Hammond-Kosack et al. [6]], although most of the $\mathrm{R}$ genes known today were isolated from model plant Arabidopsis. These tend to represent qualitative dominant resistance associated with simple Mendelian genetics. In fungal pathosystems, such genes confer resistance to biotrophs and hemi-biotrophs. Resistance to necrotrophic fungal pathogens, by contrast, is often quantitative. Not surprisingly, the genetic basis of resistance is poorly understood and relatively few resistance genes have been characterised. Those that have been reported involve specific interactions with host selective toxins (HSTs, reviewed in Wolpert et al. [7]) and are predominantly recessive in nature (i.e. susceptibility is dominant) presumably by loss or alteration of gene(s) encoding HST targets. There is evidence to suggest a HST may promote a hypersensitive-like response by acting through a resistance gene leading to cell death $[8,9]$. By contrast, of the two necrotrophic resistance genes isolated to date, the race-specific $\mathrm{Hm} 1$ detoxifying gene in maize [10] is dominant and the Asc-1 longevity assurance gene homolog in tomato $[11,12]$ is semi-dominant.

The genetic tools to clone and characterize R genes are not available in most legumes, making it difficult to study the molecular basis of resistance to diseases. Medicago truncatula, a close relative of alfalfa, is a model system and possesses a small diploid genome that is currently being sequenced, autogamous genetics and various transformation systems. The significance of $M$. truncatula lies in its susceptibility to a range of hemi-biotrophs and necro- trophs and thus is increasingly being used to study resistance to species such as Aphanomyces euteiches [13,14], Ascochyta lentis, Botrytis cinerea and B. fabae [15], Colletotrichum trifolii [16], Mycosphaerella pinodes [17], Phoma medicaginis [18], and Phytophthora medicaginis [19].

Sources of resistance to $P$. medicaginis have been previously identified among different Medicago spp. [20], and in the genetically diverse South Australian Research and Development Institute (SARDI) M. truncatula core collection [21]. Single-seeded SARDI core collection M. truncatula accessions were screened for resistance to three virulent Western Australian P. medicaginis isolates, and accession SA27063 was shown to be resistant to $P$. medicaginis OMT5, whereas A17 and SA3054 were highly susceptible [18]. In this study we describe the characterisation of the disease response in these accessions macroscopically and cytologically. The genetic basis of resistance to $P$. medicaginis in $M$. truncatula is determined together with genetic map positions for major loci conferring resistance in two different mapping populations.

\section{Results \\ Macroscopic phenotype of resistant and susceptible accessions}

Medicago truncatula SARDI core collection accessions were previously screened for their response to three $P$. medicaginis isolates. Accession SA27063 was resistant to P. medicaginis OMT5, whereas A17 and SA3054 were susceptible [18]. When leaves of three-week-old SA27063 plants were spot inoculated, the fungus was limited to the inoculation site and no chlorosis or necrosis was observed 7-10 days post infection (dpi, Figure 1a). In susceptible interactions the fungus successfully penetrated host cells and spread beyond the inoculation site, accompanied by a halo of chlorosis ahead of the infection zone. By 7-10 dpi infected leaves were entirely chlorotic or necrotic and pycnidia were apparent (Figure $1 \mathrm{~b}, \mathrm{e}$ ). Similar symptoms were observed following spray inoculation: small, microscopic hypersensitive response-like lesions and no chlorosis of leaves occurred in resistant SA27063 (10 dpi; Figure 1c); in the susceptible interactions disease symptoms were visible to the naked eye at $7 \mathrm{dpi}$. P. medicaginis colonised the surrounding plant tissue, resulting in macroscopic necrotic spots on both leaves and stems surrounded by spreading chlorosis (10 dpi; Figure $1 \mathrm{~d}$ ). As early as $10 \mathrm{dpi}$ $P$. medicaginis produced pycnidia on susceptible leaves.

\section{Microscopic disease symptoms of P. medicaginis OMT5}

Histological staining using trypan blue or DiOC6 was performed to examine differences in the infection process between resistant and susceptible accessions at the cellular level (Figures 2a-e). Following inoculation, P. medicaginis spores germinated on the leaf surface and successful pen- 


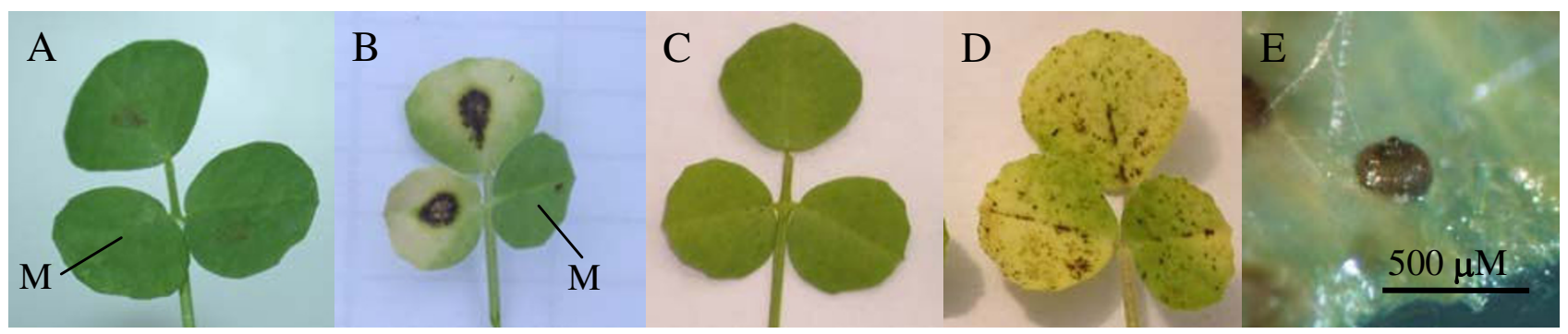

Figure I

Macroscopic disease symptoms of $\boldsymbol{P}$. medicaginis OMT5. A, SA27063 spot inoculated 10 days post inoculation (dpi). B, SA3054 spot inoculated 10 dpi. C, SA27063 spray inoculated 7 dpi. D, SA3054 spay inoculated 7 dpi. E, Pycnidium of P. medicaginis on AI7 leaf I4 dpi. M indicates mock inoculated leaflets.

etration of host cells was observed as early as 6 hours post inoculation (hpi). In both interactions, penetration attempts occurred by three routes; directly through stomata followed by penetration of the underlying mesophyll cells (Figure 2a), directly through the epidermal cells (Figure 2c), or between epidermal cells (Figure 2d). Following penetration, fungal colonisation proceeded rapidly in the susceptible accessions SA3054 and A17, whereas in the resistant accession SA27063 hyphal development was limited to individual or a few epidermal cells. Reddish-brown diaminobenzidine-tetrahydrochloride (DAB) staining to detect endogenous $\mathrm{H}_{2} \mathrm{O}_{2}$ production in cells at or around the site of penetration was observed in accession SA27063 (Figure 2b, c). However, $\mathrm{H}_{2} \mathrm{O}_{2}$ was inconsistently associated with penetration attempts and was also evident in susceptible accessions. Autofluorescence was also observed around the site of infection in both the susceptible and resistant interaction, indicating the production of phenolics (Figure $2 \mathrm{~d}, \mathrm{e}$ ).

\section{Resistance of accession SA27063 to Phoma medicaginis OMT5 in two different mapping populations}

Accession SA27063 was crossed with susceptible accessions A17 and SA3054 to create two $\mathrm{F}_{2}$ mapping populations. $\mathrm{F}_{1}$ individuals of both crosses showed $P$. medicaginis OMT5 disease symptoms equivalent to the susceptible parents, indicating recessive resistance. $F_{2}$ individuals were screened for their disease phenotype in both the SA27063 $\times$ A17 $(n=92)$ and SA27063 $\times$ SA3054 $(n=94)$ populations. Both populations differed significantly from a normal distribution $\left(P_{W}<0.0001\right.$; based on the Shapiro and Wilk test [22], which tests the null-hypothesis that a given sample derives from a normally distributed population).

The SA27063 $\times$ A17 population was not chosen for further genetic studies due to poor fertility, the presence of a reciprocal translocation between chromosomes four and eight in accession A17 [23], and a large proportion of aberrant $\mathrm{F}_{2}$ individuals (Table 1). In the SA27063 $\times$ SA3054 cross, $\mathrm{F}_{3}$ families (number of individuals per family $\geq 16$ ) were phenotyped to confirm individual $F_{2}$ phenotypes. When the same $\mathrm{F}_{3}$ families were inoculated between different experiments, mean disease scores did not alter significantly showing the phenotyping method was reliable and reproducible. The proportions of SA27063 $\times$ SA3054 $\mathrm{F}_{3}$ families distributed against the mean disease score (Figure $3 a$ ) was significantly different from a normal distribution $\left(P_{W}<0.0001\right)$. The relationship between the families' means and the variance within each family is depicted in Figure 3b. This relationship was evaluated using the Fain's test which predicts a pattern of maximum variability in intermediate families whenever at least one locus with major effect is involved [24,25]. Analysis of the $\mathrm{F}_{3}$ family disease scores using Fain's test revealed that the quadratic term was highly significant $\left(P_{/}\right.$ $t /=0.0027$; Figure $3 \mathrm{~b}$ ), indicating one or a few loci with major effects were involved in resistance to $P$. medicaginis.

\section{Genetic linkage mapping}

Genetic maps for the SA27063 $\times$ SA3054 and SA27063 $\times$ A17 mapping populations were created using both genebased and microsatellite markers. Markers were initially selected to be evenly distributed over each linkage group and were obtained from several published sources [2630]. A total of 115 markers were characterised for the SA27063 $\times$ SA3054 $(n=94)$ and 78 for SA27063 $\times$ A17 $(n$ =92) populations.

Forty-six and 58 markers for SA27063 $\times$ SA3054 and SA27063 $\times$ A17 respectively were single nucleotide polymorphisms (SNPs). The majority of these SNPs were converted to cleaved amplified polymorphic sequences [CAPS, [31]]. SNPs that could not be assayed as CAPS were genotyped using SNapShot $(\mathrm{AB})$ single fluorescent nucleotide extension. A total 53\% of markers were polymorphic between SA27063 and A17 and 35\% between SA27063 and SA3054. Marker polymorphism data, 

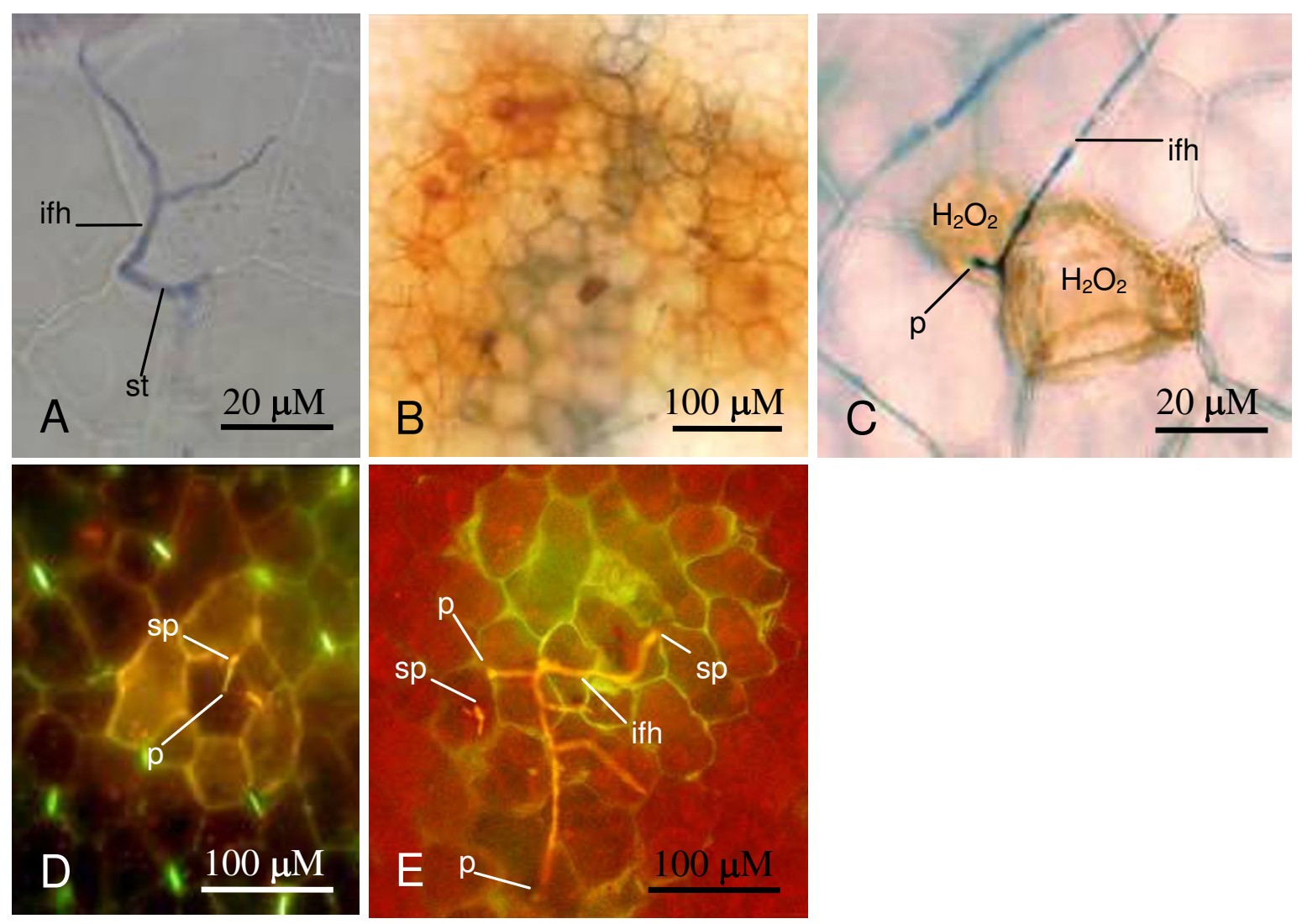

\section{Figure 2}

Microscopic analysis of $\boldsymbol{P}$. medicaginis infection of $\boldsymbol{M}$. truncatula. Leaves were spot inoculated with $P$. medicaginis OMT5 and hyphae visualised with trypan blue (A-C) or DiOC6 (D, E) at 24 and $48 \mathrm{~h}$ post infection. (A) P. medicaginis entering a stomata of SA3054. (B, C) build-up of $\mathrm{H}_{2} \mathrm{O}_{2}$ around the sites of penetration in SA27063. (D, E) Autofluorescence of phenolic compounds around the infection site in both SA27063 (D) and SA3054 (E). $\mathrm{H}_{2} \mathrm{O}_{2}=$ hydrogen peroxide; ifh = infection hyphae; $\mathrm{P}=$ point of penetration; $\mathrm{sp}=$ spore; $\mathrm{st}=$ stomata.

primer sequences, and references for SA27063 $\times$ SA3054 and for SA27063 $\times$ A17 markers have been provided [see Additional files 1 and 2].

The genetic maps for SA27063 $\times$ A17 and SA27063 $\times$ SA3054 are shown in Figures 4 and 5, respectively. As expected, the SA27063 $\times$ SA3054 map was composed of eight linkage groups, in accordance with the basic number of chromosomes in $M$. truncatula $(\mathrm{x}=8)$. The map spans a total of 488.3 centimorgans (cM) with an average distance between markers of $4.2 \mathrm{cM}$. Very few published

Table I: Fertility of $F_{2}$ progeny from crosses between AI 7 and SA27063, and SA3054 and SA27063. Figures are percentages except the average number of seeds per pod, based on 15 randomly selected pods.

\begin{tabular}{lll}
\hline & Al7 $\times$ SA27063 $n=200$ & $3054 \times$ SA27063 n=200 \\
\hline Pollen viability' & 47 & 100 \\
Av. No seeds/pod & 3.3 & 8.3 \\
Albinos & 6.2 & 0 \\
Abnormal radicals & 11.8 & 0 \\
Delayed germination ${ }^{2}$ & 4.9 & 0 \\
Dwarves & 19.3 & 0 \\
\hline
\end{tabular}

IPollen viability was determined by using Alexanders stain as described in Kamphuis et al. [23].

${ }^{2}$ Delayed germination indicates development at two weeks was equivalent to $48 \mathrm{hrs}$ in a normal seedling. 

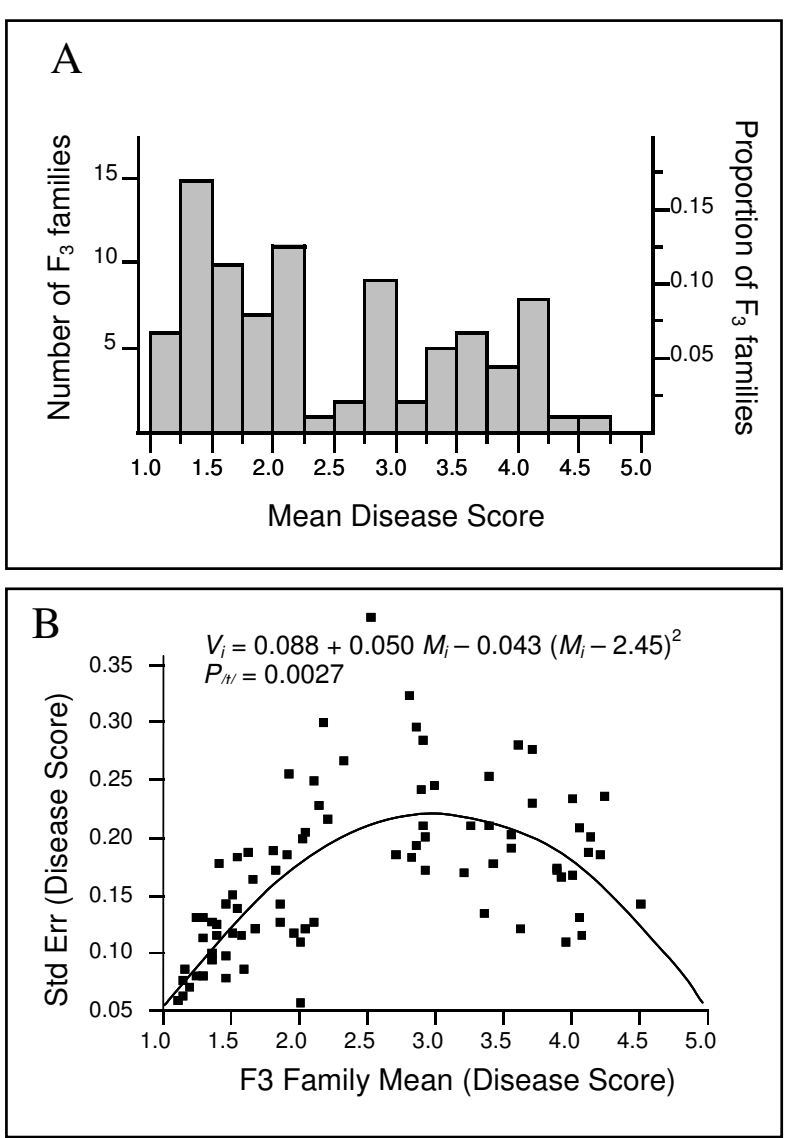

\section{Figure 3}

Response of SA27063 $\times$ SA3054 $\mathrm{F}_{3}$ families to $P$. medicaginis infection. (A) Frequency distribution of disease scores for $\mathrm{F}_{3}$ families of cross SA27063 $\times$ SA3054 infected with $P$. medicaginis OMT5 at 7 dpi. (B) Variance within a $F_{3}$ family $\left(V_{i}\right)$ as a quadratic function of its mean response $\left(M_{i}\right)$; $\left(\mathrm{P}_{|t|}\right)$ probability testing the null-hypothesis the quadratic term $(0.043)$ is significantly different from zero.

markers were polymorphic between SA27063 and SA3054 on the lower half of linkage group 2.

In contrast to the SA27063 $\times$ SA3054 map, the SA27063 $\times$ A17 map was organized into seven linkage groups, with LG4 and LG8 forming a single linkage group (Figure 4). These linkage groups have previously been reported as single linkage groups $[29,30,32]$. The establishment of new linkage relationships between LG4 and LG8 combined with observations of semisterility in hybrids involving A17 provides strong evidence that A17 bears a reciprocal translocation, which differentiates it from other M. truncatula accessions. This reciprocal translocation involves the lower arm of chromosome 4 and the lower arm of chromosome 8 [23]. All publicly available markers in the middle of LG7 were monomorphic and LG7 was therefore split into two small linkage groups named LG7a and LG7b. The SA27063 × A17 genetic map spans a total of $497.8 \mathrm{cM}$ with an average distance between markers of $6.4 \mathrm{cM}$. The linkage groups of the genetic maps presented here are similar in length to previously published maps $[29,30]$ with the exception of LG3. In SA27063 $\times$ SA3054 the distance between DK501R and CysPR1 was $50.9 \mathrm{cM}$, whereas in SA27063 $\times$ A17 this distance was $79.6 \mathrm{cM}$.

\section{QTL mapping of spring black stem and leaf spot resistance in $M$. truncatula}

Two major quantitative trait loci (QTLs) for resistance to P. medicaginis OMT5 were identified, one in each mapping population. In SA27063 $\times$ A17, the effect of the QTL $(d=$ 1.4) was significantly different from zero $(P<0.00001$; LOD = 7.37). The locus was named resistance to the necrotroph Phoma medicaginis one (rnpm1) and was located on LG4 (Figure 4), tightly linked to AW256637. rnpm1 explains $33.6 \%$ of the phenotypic variance in the population's response to infection by $P$. medicaginis OMT5, based on a 1-5 scale [18]. In SA27063 $\times$ SA3054, the QTL effect $(d=1.46)$ was significantly different from zero $(P<0.00001 ;$ LOD $=6.77)$. This locus was named resistance to the necrotroph Phoma medicaginis two (rnpm2) and was located on LG8 (Figure 5), tightly linked to MtB262. rnpm2 explains $29.6 \%$ of the phenotypic variance. In SA27063 × SA3054, a second QTL, rnpm3 was located on linkage group one. This locus is statistically less significant $(P=0.04 ;$ LOD $=3.37)$, has a smaller effect $(d$ $=1.1$ ), and explains $19.4 \%$ of the total phenotypic variance.

\section{Fine mapping rnpm2}

To initiate fine mapping of rnpm2 on the long arm of chromosome eight, an additional $434 \mathrm{~F}_{2}$ individuals (524 in total) were screened for recombination within a $8.2 \mathrm{cM}$ interval between markers MtB294, MtB174 and MtB139 (Figure 5). $\mathrm{F}_{3}$ families from the selected recombinant $\mathrm{F}_{2}$ individuals were phenotypically characterised. As mentioned above, locus rnpm2 explains $29.6 \%$ of the total variance in the population's response to the fungal infection when measured in a 1-5 scale. However, recombinant families that showed the SA27063-like (resistant) phenotype correlated with homozygous SA27063 alleles at rnpm 2 in $78.5 \%$ of instances. The resistant parent consistently showed little or no visible necrotic symptoms, while disease progression in the susceptible parent was variable and ranged from $2.5-4.5$. The variance in disease scores was therefore partly a function of the continuum of mean quantitative susceptible disease scores and the sum of disease scores in heterozygous $\mathrm{F}_{3}$ families. Recombination break point analysis was therefore used to fine map rnpm2, with phenotyping data from thirty-eight informative $\mathrm{F}_{3}$ families in conjunction with further polymorphic markers to resolve recombination break points. The loca- 


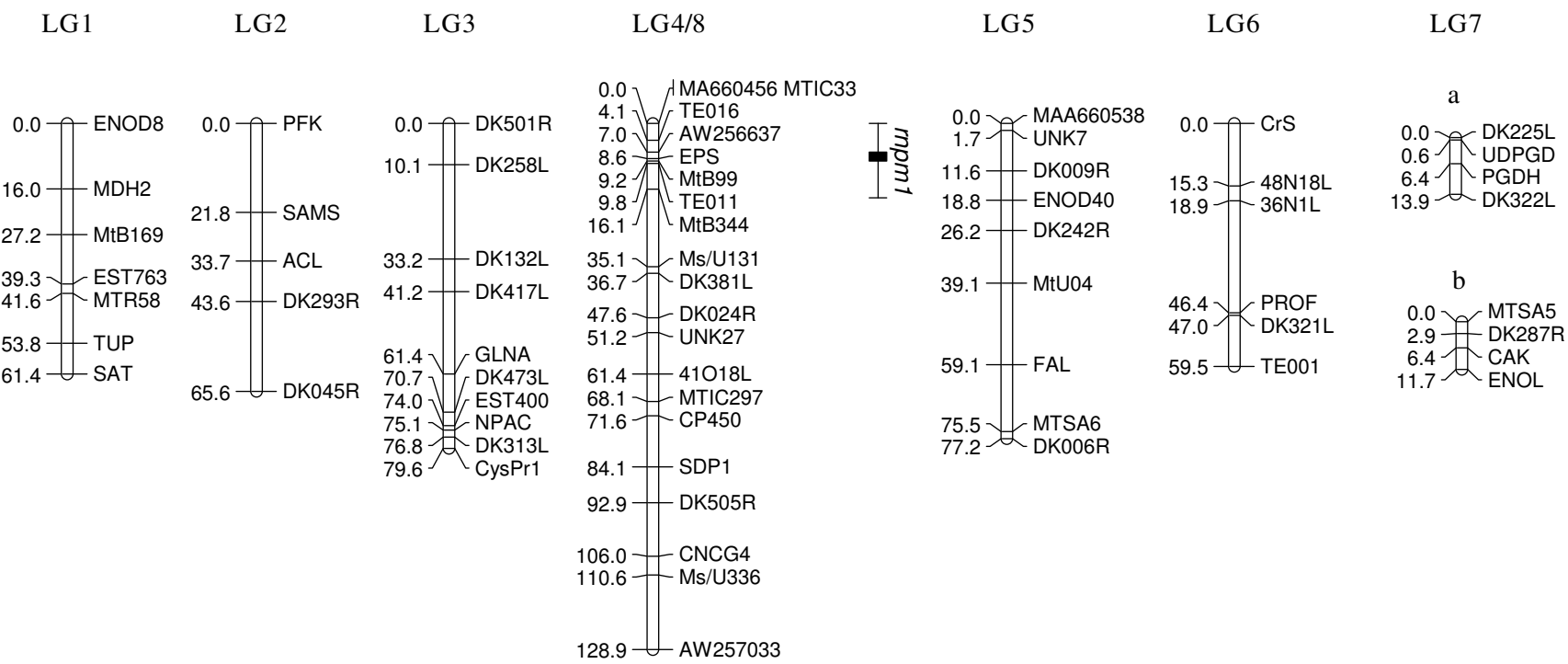

\section{Figure 4}

Genetic map of $M$. truncatula constructed using accessions SA27063 and AI 7, based on $92 F_{2}$ individuals. The genetic map comprises 78 markers and spans $497.8 \mathrm{cM}$. Horizontal lines show marker positions. Horizontal lines show marker positions and marker names are located to the right. Genetic distances (cM) are located to the left of each marker. The genomic location of the QTL rnpm I for P. medicaginis resistance is depicted to the right of LG4, with the standard deviation of its location depicted by lines either side.

tion of rnpm2 was mapped to a $0.8 \mathrm{cM}$ interval between markers h2_16a6a and h2_21h11d (Figure 6).

\section{Discussion and Conclusions}

The main purpose of this research was to examine differences in disease phenotypes and the genetic basis of resistance to $P$. medicaginis in $M$. truncatula. The resistant phenotype was characterized macroscopically by the presence of small hypersensitive response-like spots, whereas susceptible accessions showed typical stem and leaf lesions, within which pycnidia, or fruiting bodies, formed. In the resistant accession SA27063 pycnidia were not apparent, or where present they developed following a delay of 2-3 weeks, predominantly on senescing leaves. Microscopically, no differences were observed in penetration attempts between resistant and susceptible accessions, and no obvious changes uniquely associated with resistance were evident with the exception of fungal growth being restricted to one or a few plant cells. Production of $\mathrm{H}_{2} \mathrm{O}_{2}$ was observed in resistant SA27063-infected leaves but was also detectable in susceptible SA3054 and A17. $\mathrm{H}_{2} \mathrm{O}_{2}$ has been reported to play an important role in resistance involving a hypersensitive response [33,34]. Build up of reactive oxygen species (ROS) is generally observed following penetration by fungi with a hemibiotrophic or biotrophic lifestyle [16,35] and subsequently leads to resistance against such fungi, while necrotrophic pathogens may stimulate ROS production and subse- quent cell death to facilitate subsequent infection [36]. Examination of inoculated leaf tissue also showed accumulation of autofluorescent phenolic compounds around the infection site in both resistant and susceptible interactions. Phenolic compounds, such as flavonoids and isoflavonoids, are an important aspect in legume plant defence [37], and there is ample evidence for their accumulation in response to fungal pathogens in Medicago species [38-42]. Pilot transcriptional profiling at $12 \mathrm{hpi}$ using the Mt16kOLI1plus microarray [43] showed significant changes in induced genes related to oxidative burst, cell wall strengthening, lipid metabolism, and the phenylpropanoid pathway leading to isoflavonoid production (L. Kamphuis, unpublished data). However, similar levels of induction occurred in both the resistant and susceptible interactions. Cytological similarities reported here are supported in oats, where cell death and induction of defence-related responses were observed in response to the HST victorin in sensitive genotypes (reviewed by Sweat et al., 2007 [9]), and in barley where a susceptiblespecific second ROS burst was observed in the later stages of the infection [44]. Detailed expression and metabolic profiling may help to identify differences in the HR response and the abundance and composition of defencerelated compounds and their role in restricting $P$. medicaginis colonisation. 
LG1

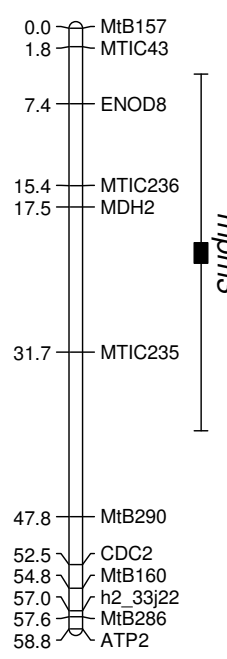

LG2

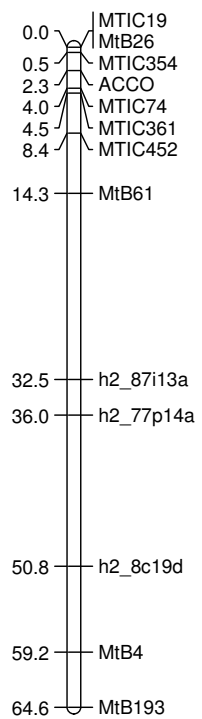

LG3

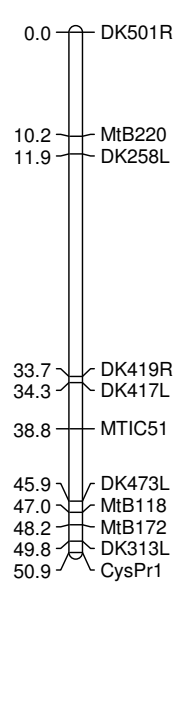

LG4

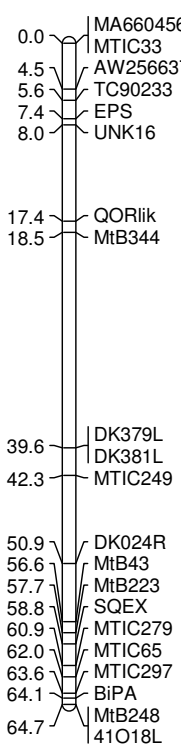

LG5

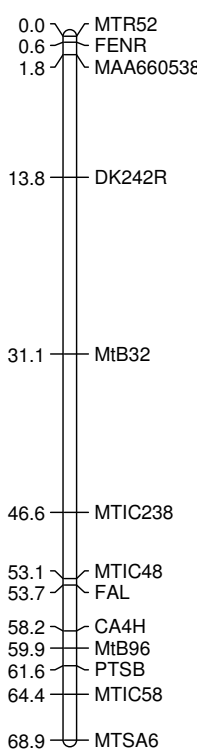

LG6

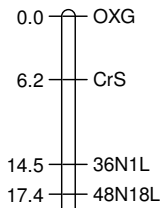

LG7

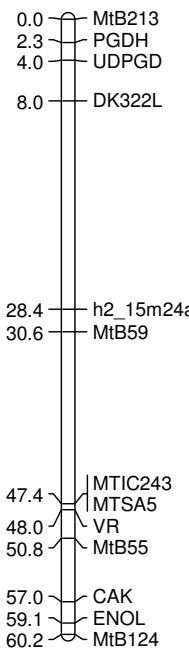

LG8

\section{Figure 5}

Genetic map of $M$. truncatula constructed using accessions SA27063 and SA3054, based on $94 F_{2}$ individuals. The genetic map comprises II 5 markers and spans $488.3 \mathrm{cM}$. Horizontal lines show marker positions and marker names are located to the right. Genetic distances (cM) are located to the left of each marker. The genomic locations of QTLs rnpm2 and rnpm3 for P. medicaginis resistance are depicted the right of LG8 and LGI respectively, with standard deviations depicted by lines either side.

The lower proportion of polymorphic makers in the SA27063 $\times$ SA3054 cross suggested this is a narrower cross than SA27063 $\times$ A17. The total map length for SA27063 $\times$ SA3054 $(488.3 \mathrm{cM})$ was smaller than previously reported genetic maps $[29,30,32]$ and in the SA27063 $\times$ A17 map. The genetic distance between markers DK501R and CysPR1 on LG3 in particular was notably smaller than in the SA27063 × A17 map. LG3 corresponds with chromosome three, the largest chromosome of $M$. truncatula [45]. One explanation for this phenomenon could be that accessions SA27063 and SA3054 are sympatric [21], and may share long stretches of homology in chromosome three, resulting in a lack of detectable recombination.

Quantitative trait loci (QTL) analysis is often used to identify and characterise loci conferring resistance. QTL mapping allows the roles of specific $R$-loci to be described, race-specificity of partial resistance genes can be assessed, and interactions between resistance genes, plant development and the environment to be analysed [46]. QTL analysis for $P$. medicaginis resistance in the SA27063 $\times$ A17 mapping population revealed a QTL on chromosome four $(r n p m 1)$ and in the SA27063 $\times$ SA3054 population a QTL on chromosome eight ( $r n p m 2)$, both recessive in nature. As explained in the results, although the rnpm2 QTL explained a relatively small amount of the total calculated variance for resistance $(29.6 \%)$, SA27063-like or resistant individuals in the $\mathrm{F}_{2}$ mapping population correlated with individuals homozygous for SA27063 alleles at rnpm2 in approx. $80 \%$ of instances. Among the $\mathrm{F}_{3}$ families used in fine mapping and predicted to be resistant by their genotype, this proportion remained constant, allowing rnpm2 to be mapped to a $0.8 \mathrm{cM}$ interval between markers h2_16a6a and h2_21h11d by recombination break point analysis. The proportion of resistant homozygous SA27063 rnpm2 individuals suggested $r n p m 2$ is epistatic to one or several cis- or trans-acting genes or regulatory elements, possibly locus rnpm3 on LG1. As the detection and accuracy of minor QTLs in segregating populations largely depends on map quality and population size, increasing the initial mapping population size or genome-wide analysis of expressed QTLs [47] may reveal other minor QTLs and will enable thorough examination of the interaction between rnpm2 and rnpm3.

We did not proceed with fine mapping rnpm1 in the SA27063 $\times$ A17 cross, due to poor fertility and a large proportion of aberrant phenotypes among the $F_{2}$ progeny (Table 1), which may cause biased representation of particular gamete genotypes. Furthermore, accession A17 


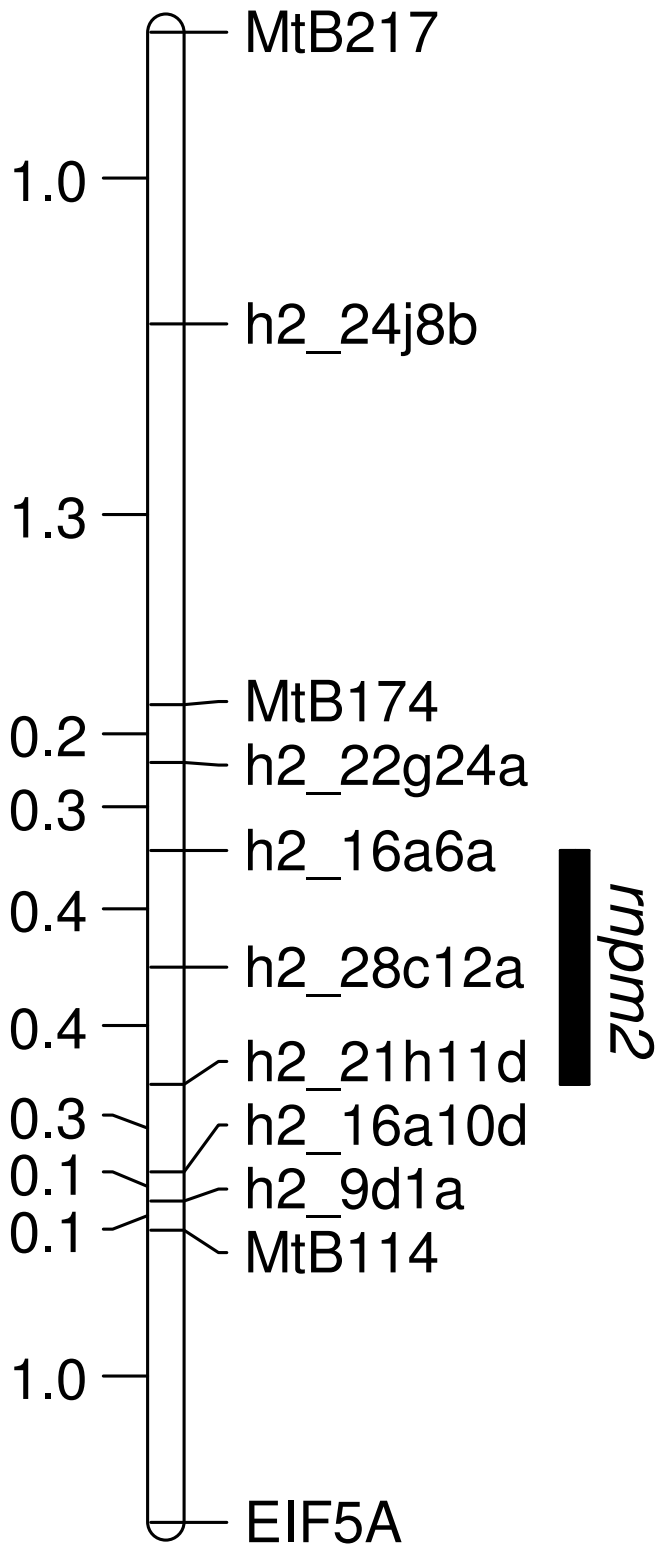

Figure 6

Fine map genetic position of rnpm2. The genomic region encompassing rnpm2 for $P$. medicaginis resistance in the SA27063 $\times$ SA3054 population is depicted in a $0.8 \mathrm{cM}$ interval between markers h2_I6a6a and h2_2I hI Id on lower LG8. The location is based on a recombination point analysis using recombinant $F_{3}$ families selected from $528 F_{2}$ individuals.

bears a reciprocal translocation, involving chromosomes four and eight, and exhibits reduced pollen viability [23]. All previously published $M$. truncatula genetic maps used accession A17 or a closely related line, J6, as one of the parents. The SA27063 $\times$ SA3054 genetic map is the first map produced in M. truncatula not involving A17 as a parental line and is therefore useful in identifying the correct position of ambiguously placed markers in A17derived genetic maps. The reciprocal translocation involves markers distal to rnpm 2 at approximately $30 \mathrm{cM}$ or less and is therefore unlikely to affect the detection of this QTL among SA27063 × A17 progeny.

The simplest explanation for the existence of two separate QTLs among progeny in two crosses involving the same resistant parent is that genotype-specific susceptibility loci may interact with undetermined $P$. medicaginis OMT5 virulence factors or HSTs. The rnpm1 locus is tightly linked to marker AW256637 on BAC AC144658, which is in a contig containing a cluster of TIR-NBS-LRR and disease resistance protein-like genes [48], while no RGAs are apparent in the genomic sequence of the reference accession A17 at the rnpm2 locus. The recessive host genotype-specific nature of rnpm1 and rnpm2 bears similarities to the racespecific Pyrenophora tritici-repentis toxin insensitivity QTLs in wheat [49], where multiple, isolate-specific HSTs interact with a given host genotype to produce a susceptible phenotype. To date, no $P$. medicaginis phytotoxins have been characterised in vivo during infection. However, the presence of chlorotic symptoms in susceptible $M$. truncatula accessions inoculated with $P$. medicaginis in advance of the fungal hyphae, and the large number of toxins produced by other Phoma species suggests that susceptible plants with Rnpm1 and Rnpm2 are sensitive to a toxin and variation in these genes ( $r n p m 1$ and $r n p m 2)$ results in lack of sensitivity.

Future work will be directed at further fine mapping and isolation of $r n p m 2$, in conjunction with gene expression studies to identify genes controlling resistance and candidates for rnpm 2 in the region of interest. In addition, the possible involvement of fungal phytotoxins during infection will be characterised and their interaction with the resistance QTL evaluated.

\section{Methods}

\section{Growth conditions and mapping populations}

To ensure even germination, seeds were scarified with sand using a mortar and pestle, transferred to a Petri dish lined with blotting paper, and irrigated with sterile water. The seeds were kept at $4^{\circ} \mathrm{C}$ for $48 \mathrm{~h}$, and germinated at room temperature overnight. Seedlings were sown in vermiculite (Richgro Garden Products, Jandakot, Western Australia 6164), fertilized twice a week using Optigrow fertilizer (Growth Technology, O'Connor, Western Australia 6163) and grown under natural light in a temperature controlled greenhouse $\left(22^{\circ} \mathrm{C}\right.$ day, $18^{\circ} \mathrm{C}$ night $)$.

Parental M. truncatula accessions were identified from the disease phenotype screens described by as being either 
resistant (SA27063) or susceptible (SA3054 and A17) to P. medicaginis OMT5 [18]. Crosses between SA27063 and either A17 or SA3054, as pollen donors, were obtained by a manual crossing procedure [32]. $\mathrm{F}_{1}$ seeds were collected and heterozygosity verified with genetic markers polymorphic between the parental accessions.

\section{Inoculation procedures and resistance evaluation}

$P$. medicaginis OMT5 inoculum was prepared by growing the isolate on wheat meal agar plates (WMA, 12 g ground wheat meal, $12 \mathrm{~g}$ agar, and 1 litre of distilled water). The plates were incubated at $22^{\circ} \mathrm{C}$ for $28-42$ days under U/V. Conidia were harvested by incubating plates with $10 \mathrm{ml}$ milliQ water for $20 \mathrm{~min}$. and the suspension filtered through a glasswool syringe. Inoculations of were performed at the fourth trifoliate stage. Two methods of inoculation were used: spraying with an artists airbrush to runoff $\left(2 \times 10^{6}\right.$ spores/ml, $0.05 \%$ Tween 20$)$, using a rotating platform to ensure an even distribution over plant surfaces; and spot inoculation with $10 \mu \mathrm{l}$ droplets of spore suspension $\left(1 \times 10^{6}\right.$ spores $/ \mathrm{ml}, 0.05 \%$ Tween 20$)$ on five leaves per plant. These were the unifoliate, two leaflets of the first trifoliate, and two leaflets of the second trifoliate. The third leaflets of the second and third trifoliates were mock inoculated. To ensure high humidity to stimulate conidia germination, plants were placed in sealed propagator trays for 48 hours.

$\mathrm{F}_{2}$ individuals were spot inoculated and macroscopically evaluated at 7 days post inoculation (dpi), then rescored at $10 \mathrm{dpi}$ to confirm more resistant disease reactions as on a 1 (resistant) - 5 (susceptible) scale described by Ellwood et al. [18]. $\mathrm{F}_{3}$ families were spray inoculated using a minimum of $16 \mathrm{~F}_{3}$ individuals per family and a randomised plot design with two representative $F_{3}$ individuals per family per plot. Disease symptoms were evaluated at 7 and $10 \mathrm{dpi}$ according to the 1-5 scale devised by Salter and Leath [50]. All inoculations incorporated parental control accessions.

\section{Cytology of the M. truncatula-P. medicaginis pathosystem}

Three different staining methods were used to study the M. truncatula - P. medicaginis interaction microscopically:

1) Superficial fungal growth was observed by staining with fluorescent dye 3'3-dihexyloxacarbocyanine iodide (DiOC6 $_{(3)}$, Sigma-Aldrich). Whole or part-leaf samples were immersed in fresh aqueous solutions of the fluorescent dye $3 \mathrm{DiOC6}_{(3)}$ at $50 \mathrm{mg} \mathrm{mL}^{-1}$, prepared from $\mathrm{DiOC6}_{(3)}$ stock solution in ethanol $\left(0.5 \mathrm{mg} \mathrm{mL}^{-1}\right.$, stored at $-20^{\circ} \mathrm{C}$ as described by Duckett and Read [51]. Between 2-3 minutes of exposure normally gave an adequate level of stain absorption. The samples were then placed on slides and the excess stain solution drawn off gently from the edges with tissue. The samples were examined under UV with a light fluorescent Olympus $\mathrm{BH}-2$ microscope fitted with an epifluorescence filter B2A (450-490 nm excitation filter and a $520 \mathrm{~nm}$ barrier filter). Hyphae stained bright yellow, conidiospores stained bright green to yellow with increasing age, plant cells exhibiting a hypersensitive response were dark brown or dark green, and healthy cells appeared a deep red colour due to the autofluorescence of intact chloroplasts. Yellow autofluorescence of phenolic compounds could also be observed under UV-light.

2) Superficial and intracellular fungal growth was observed using trypan blue staining. Leaves were fixed in Farmers Fluid until completely cleared. The cleared leaves were immersed in a $0.03 \%$ trypan blue stain concentrate mixed with equal volume of $100 \%$ ethanol for $30 \mathrm{~min}$ utes, then destained in $2.5 \mathrm{~g} / \mathrm{mL}$ chloral hydrate and examined under a Olympus $\mathrm{BH}-2$ light-microscope.

3) To visualize production of $\mathrm{H}_{2} \mathrm{O}_{2}$ in response to $P$. medicaginis penetration, a DAB (Diaminobenzidine-tetrahydrochloride) staining solution was used [34]. The petioles were placed in the DAB staining solution $(1 \mathrm{mg} \mathrm{DAB} / \mathrm{mL}$ $\mathrm{H}_{2} \mathrm{O}$ pH 3.8, Sigma) for 48 hours. The leaves were fixed and cleared in a solution of ethanol: chloroform (v/v 4:1) and $0.15 \%$ TCA for 2-3 hours. After DAB staining leaves were stained with Trypan Blue staining solution for 5-10 minutes. The stained leaves were examined under an Olympus BH-2 light-microscope.

\section{Statistical analysis of the disease resistance data}

Departure from normality for the distribution of disease scores was tested using the $W$ test of Shapiro and Wilk [22]. Fain's test was used to evaluate the relationship between the means of the F3 families and the variance within the families [25]. This test assumes that if resistance is determined by one or a few genes with a large effect, that families possessing the most extreme phenotypes are likely to be homozygous, exhibiting low variance within the family. Families with intermediate phenotypes are more likely to be heterozygous, exhibiting large variances (for resistance) within each family. The relationship between families can be described using a quadratic equation, where a significant value $\left(P_{|t|}<0.05\right)$ indicates the presence of one (or a few) major genes. All the statistical analysis of the data described above was carried out using JMP-IN 5.1 software (SAS Institute, Cary, NC).

\section{Identification of polymorphic DNA markers}

Different types of PCR markers were used to identify polymorphic markers; EST-based intron targeted markers [30]; microsatellites located in introns [28]; microsatel- 
lites from sequenced BACs [52] and other published sources $[26,29]$.

Temperature gradient PCR was used to identify the optimum annealing temperature for each primer pair using the standard reference accession $M$. truncatula A17 as a positive control. The following basic PCR protocol was used with minor variations: 50 ng of genomic DNA template, $1 \times$ PCR reaction buffer, $2 \mathrm{mM} \mathrm{MgCl} 2,0.25 \mathrm{mM}$ of each dNTP, $10 \mathrm{pmol}$ of each primer and 1 unit of Taq DNA polymerase. Thermocycling conditions (with minor variations) were: 2 minutes initial denaturation at $94^{\circ} \mathrm{C}$ followed by 36 cycles of $94^{\circ} \mathrm{C}$ for $30 \mathrm{sec}$, marker-specific annealing temperatures for $30 \mathrm{sec}, 72^{\circ} \mathrm{C}$ for $60 \mathrm{sec}$, then a final extension step of $5 \mathrm{~min}$ at $72^{\circ} \mathrm{C}$. Where no length or published restriction enzyme polymorphisms were available, PCR products with a single amplicon were direct sequenced using BigDye 3.1 Terminator Cycle Sequencing Ready Reaction Mix (Applied Biosystems [AB], Foster City, California) and the products run on an $A B$ Prism 3730 DNA sequencer. Polymorphisms in the DNA sequences were identified by aligning the sequences in Vector NTI Suite 9.0 (Invitrogen, Carlsbad, California). Where restriction enzymes recognising differences in DNA sequence were available, markers were run as co-dominant CAPS [31]. Single Nucleotide Polymorphisms (SNPs) for which no restriction enzyme was available were detected using SNaPshot (AB), and analysed using GeneMapper v. 3.7 (AB). Large fragment size polymorphisms were resolved by agarose gel electrophoresis and visualised using ethidium bromide staining. Small fragment size polymorphisms were resolved by native polyacrylamide gel electrophoresis or by $\mathrm{AB}$ Prism 3730 capillary sequencer using fluorescently labelled primers and a GeneScan ${ }^{\mathrm{TM}} 500$ LIZ $^{\circledast}$ Size Standard (AB).

\section{Genotyping and linkage analysis}

Genomic DNA from the parents and $\mathrm{F}_{2}$ individuals were extracted as previously described [21] and polymorphic markers genotyped as described above. A Pearson Chisquare analysis was applied to test the observed segregation ratio of parental alleles against the expected Mendelian segregation ratio for co-dominant inheritance in a $\mathrm{F}_{2}$ population, $1: 2: 1$, to remove highly distorted markers. Marker order and map distances were calculated with Multipoint v1.2 software [53] using a maximum recombination fraction (rf) of 0.210 and the Kosambi mapping function. To test the stability of the order of markers for each linkage group (LG), a Jackknife re-sampling approach was used with 5000 iterations. Markers that violated monotonic increase of rfs (i.e. deviation from the expected increase of rf between a marker and its subsequent neighbours) were detected and removed using the control of monotony function. Marker orders were accepted if the probability was greater than 0.90 .
Removed markers were re-attached to the genetic map in the interval of most probable fit (e.g. minimum increase in the number of recombination events).

\section{QTL analysis}

QTL analysis was performed with MultiQTL v2.5 [53] by applying a general interval mapping and marker restoration method as described by Lichtenzveig et al. [54]. The hypotheses that a single locus or two linked loci have an effect on resistance to $P$. medicaginis were evaluated. Firstly, 5000 permutation tests were performed on the hypothesis that one locus on a chromosome has an effect on the disease resistance $\left(\mathrm{H}_{1}\right)$ versus the null hypothesis $\left(\mathrm{H}_{0}\right)$ that the locus has no effect on the disease resistance. Secondly, 3000 permutation tests were run on the hypothesis that a single locus has an effect on disease resistance versus two linked loci. The model with the highest LOD score was fitted to the QTL and when the models did not differ significantly the simpler model was chosen ('one locus-one trait'). Five thousand bootstrap samples were run to assess the estimates and the standard deviation (SD) of the main parameters: locus effect, its chromosomal position, its LOD score and the proportion of explained variability (PEV).

\section{Authors' contributions}

SE and RPO designed research. LK and SE conducted disease screens, phenotyping, polymorphism discovery, genotyping and genetic mapping. JL provided advice on statistical analyses. LK, JL and SE analysed the data.

\section{Additional material}

\section{Additional file 1}

PCR markers used to generate a genetic map in an $F_{2}$ population between accessions SA27063 and SA3054

Click here for file

[http://www.biomedcentral.com/content/supplementary/14712229-8-30-S1.doc]

\section{Additional file 2}

PCR markers used to generate a genetic map in an $F_{2}$ population between accessions SA27063 and A17

Click here for file

[http://www.biomedcentral.com/content/supplementary/14712229-8-30-S2.doc]

\section{Acknowledgements}

This research was funded by the Grains Research and Development Corporation of Australia (GRDC). We thank Megan Jordan and Angela Williams for technical assistance.

\section{References}

I. Nutter FR, Guan J, Gotlieb AR, Rhodes LH, Grau CR, Sulc RM: Quantifying alfalfa yield losses caused by foliar diseases in 
lowa, Ohio, Wisconsin, and Vermont. Plant Dis 2002, 86(3):269-277.

2. Crawford EJ, Lake AWH, Boyce KG: Breeding annual Medicago species for semi-arid conditions in southern Australia. Adv Agron 1989, 42:399-437.

3. Barbetti MJ: Fungal foliage diseases of pasture legumes. J Agric (W Aust) 1983, I:10-12.

4. Barbetti MJ, Nichols PGH: Effect of Phoma medicaginis and Leptosphaerulina trifolii on herbage and seed yield and coumestrol content of annual Medicago species. Phytophylactica 1991, 23:223-227.

5. Barbetti MJ: Resistance in annual Medicago species to Phoma medicaginis and Leptosphaerulina trifolii on herbage under field conditions. Aust J Expt Agric 1995, 35:209-2।4.

6. Hammond-Kosack KE, Parker JE: Deciphering plant-pathogen communication: fresh perspectives for molecular resistance breeding. Curr Opin Biotechnol 2003, I4(2): 177-193.

7. Wolpert T], Dunkle LD, Ciuffetti LM: Host-selective toxins and avirulence determinants: What's in a name? Annual Review of Phytopathology 2002, 40(I):25I-285.

8. Mayama S, Bordin APA, Morikawa T, Tanpo H, Kato H: Association of avenalumin accumulation with co-segregation of victorin sensitivity and crown rust resistance in oat lines carrying the Pc-2 gene. Physiol Mol Plant Pathol 1995, 46(4):263-274.

9. Sweat TA, Wolpert TJ: Thioredoxin $h 5$ is required for victorin sensitivity mediated by a CC-NBS-LRR gene in Arabidopsis. Plant Cell 2007, 19(2):673-687.

10. Johal GS, Briggs SP: Reductase activity encoded by the HMI disease resistance gene in maize. Science 1992 258(5084): $985-987$.

II. Brandwagt BF, Mesbah LA, Takken FLW, Laurent PL, Kneppers TJA, Hille J, Nijkamp HJJ: A longevity assurance gene homolog of tomato mediates resistance to Alternaria alternata f. sp. lycopersici toxins and fumonisin BI. Proceedings of the National Academy of Sciences 2000, 97(9):496I-4966.

12. Clouse SD, Gilchrist DG: Interaction of the asc locus in F8 paired lines of tomato with Alternaria alternata f. sp. lycopersici and AAL-toxin. Phytopathology 1987, 77:80-82.

13. Colditz F, Braun HP, Jacquet C, Niehaus K, Krajinski F: Proteomic profiling unravels insights into the molecular background underlying increased Aphanomyces euteiches-tolerance of Medicago truncatula. Plant Mol Biol 2005, 59(3):387-406.

14. Vandemark G, Grunwald N: Reaction of Medicago truncatula to Aphanomyces euteiches Race 2. Archives of Phytopathology and Plant Protection 2004, 37:59-67.

15. Ellwood S, Kamphuis LG, Pfaff T, Oliver RP, Samac DA, Foster-Hartnett D, Tivoli B, Onfroy C, Moussart A, Villegas AM, Sillero JC, Rubiales D: Inoculation and growth with foliar pathogenic fungi. Medicago truncatula Handbook 2006 [http://www.noble.org/Medicago Handbook/pdf/InoculationGrowth FoliarPathogenic.pdf]. Ardmore , The Samuel Roberts Noble Foundation

16. Torregrosa C, Cluzet S, Fournier J, Huguet T, Gamas P, Prosperi JM, Esquerre-Tugaye MT, Dumas B, Jacquet C: Cytological, genetic and molecular analysis to characterize compatible and incompatible interactions between Medicago truncatula and Colletotrichum trifolii. Mol Plant Microbe Interact 2004, I 7(8):909-920.

17. Moussart A, Onfroy C, Lesne A, Esquibet M, Grenier E, Tivoli B: Host status and reaction of Medicago truncatula accessions to infection by three major pathogens of pea (Pisum sativum) and alfalfa (Medicago sativa). Eur J Plant Pathol 2007, I I 7(I):57-69.

18. Ellwood SR, Kamphuis LG, Oliver RP: Identification of sources of resistance to Phoma medicaginis isolates in Medicago truncatula SARDI core collection accessions, and multigene differentiation of isolates. Phytopathology 2006, 96:1330-1336.

19. Moussart A, Tivoli B, Samac DA, D'Souza N: Medicago truncatula resistance to Oomycetes. Medicago truncatula Handbook 2006 [http://www.noble.org/MedicagoHandbook/pdf/OomycetesResist ance.pdf]. Ardmore, The Samuel Roberts Noble Foundation

20. O'Neill NR, Bauchan GR, Samac DA: Reactions in the annual Medicago spp. core germ plasm collection to Phoma medicaginis. Plant Dis 2003, 87(5):557-562.

21. Ellwood SR, D'Souza NK, Kamphuis LG, Burgess TI, Nair RM, Oliver RP: SSR analysis of the Medicago truncatula SARDI core collection reveals substantial diversity and unusual genotype dispersal throughout the Mediterranean basin. Theor Appl Genet 2006, I I 2(5):977-983.

22. Shapiro SS, Wilk MB: An analysis of variance test for normality (complete samples). Biometrika 1965, 52(3 and 4):59|-6II.

23. Kamphuis LG, Williams AH, D'Souza NK, Pfaff T, Ellwood SR, Groves EJ, Singh KB, Oliver RP, Lichtenzveig J: The Medicago truncatula reference accession $A 17$ has an aberrant chromosomal configuration. New Phytol 2007, 174(2):299-303.

24. Lichtenzveig J, Shtienberg D, Zhang HB, Bonfil DJ, Abbo S: Biometric analyses of the inheritance of resistance to Didymella rabiei in chickpea. Phytopathology 2002, 92(4):417-423.

25. Lynch M, Walsh B: Genetics and analysis of quantitative traits. Sunderland, USA, Sinauer Associates; 1997.

26. Baquerizo-Audiot E, Desplanque B, Prosperi JM, Santoni S: Characterization of microsatellite loci in the diploid legume Medicago truncatula (barrel medic). Mol Ecol Notes 200I:I-3.

27. Choi HK, Mun JH, Kim DJ, Zhu H, Baek JM, Mudge J, Roe B, Ellis N, Doyle JJ, Kiss GB, Young ND, Cook DR: Estimating genome conservation between crop and model legume species. PNAS 2004, I 0 I(43): I 5289-15294.

28. Gutierrez MV, Vaz Patto MC, Huguet T, Cubero JI, Moreno MT, Torres AM: Cross-species amplification of Medicago truncatula microsatellites across three major pulse crops. Theor Appl Genet 2005, I I 0(7): 1210-1217

29. Mun JH, Kim DJ, Choi HK, Gish J, Debellé F, Mudge J, Denny R, Endré S, Saurat O, Dudez AM, Kiss GB, Roe B, Young ND, Cook DR: Distribution of microsatellites in the genome of Medicago truncatula: A resource of genetic markers that integrate genetic and physical maps. Genetics 2006, I72:254I-2555.

30. Choi HK, Kim D, Uhm T, Limpens E, Lim H, Mun JH, Kalo P, Penmetsa RV, Seres A, Kulikova O, Roe BA, Bisseling T, Kiss GB, Cook DR: A sequence-based genetic map of Medicago truncatula and comparison of marker colinearity with $M$. sativa. Genetics 2004, I66(3): I 463-I502.

31. Konieczny A, Ausubel FM: A procedure for mapping Arabidopsis mutations using co-dominant ecotype-specific PCR-based markers. Plant Journal 1993, 4(2):403-4I0.

32. Thoquet P, Gherardi M, Journet EP, Kereszt A, Ane JM, Prosperi JM, Huguet $T$ : The molecular genetic linkage map of the model legume Medicago truncatula: an essential tool for comparative legume genomics and the isolation of agronomically important genes. BMC Plant Biol 2002, 2(I):I EPub.

33. Huckelhoven R, Dechert $\mathrm{C}$, Kogel $\mathrm{KH}$ : Non-host resistance of barley is associated with a hydrogen peroxide burst at sites of attempted penetration by wheat powdery mildew fungus. Molecular Plant Pathology 200I, 2(4): 199-205

34. Huckelhoven R, Fodor J, Preis C, Kogel KH: Hypersensitive cell death and papilla formation in barley attacked by the powdery mildew fungus are associated with hydrogen peroxide but not with salicylic acid accumulation. Plant Physiol 1999. I I 9:125I-1260.

35. Glazebrook J: Contrasting mechanisms of defence against biotrophic and necrotrophic pathogens. Annual Review of Phytopathology 2005, 43(I):205-227

36. Torres MA, Jones JDG, Dangl JL: Reactive oxygen species signaling in response to pathogens. Plant Physiol 2006, I 4 I (2):373-378.

37. Dixon RA: Natural products and plant disease resistance. Nature 200 I, 4 I I:843-847.

38. Deavours BE, Liu C], Naoumkina MA, Tang Y, Farag MA, Sumner LW Noel JP, Dixon RA: Functional analysis of members of the isoflavone and isoflavanone O-methyltransferase enzyme families from the model legume Medicago truncatula. Plant Mol Biol 2006, 62:715-733.

39. Dixon RA, Achnine L, Kota P, Liu CJ, Reddy MSS, Wang L: The phenylpropanoid pathway and plant defence - a genomics perspective. Molecular Plant Pathology 2002, 3(5):37I-390.

40. He XZ, Dixon RA: Genetic manipulation of isoflavone 7-Omethyltransferase enhances biosynthesis of 4'-O-methylated isoflavonoid phytoalexins and disease resistance in alfalfa. Plant Cell 2000, I 2(9): 1689-1702.

4I. Liu CJ, Huhman D, Sumner LW, Dixon RA: Regiospecific hydroxylation of isoflavones by cytochrome P450 8IE enzymes from Medicago truncatula. Plant Journal 2003, 36:47| - 484 .

42. Barbetti MJ: Resistance in annual Medicago spp. to Phoma medicaginis and Leptosphaerulina trifolii and its relationship 
to induced production of a phytoestrogen. Plant Dis 2007, 9I(3):239-244.

43. Kuster H, Hohnjec N, Krajinski F, El Yahyaoui F, Manthey K, Gouzy J, Dondrup M, Meyer F, Kalinowski J, Brechenmacher L, van Tuinen D, Gianinazzi-Pearson V, Puhler A, Gamas P, Becker A: Construction and validation of cDNA-based Mt6k-RIT macro- and microarrays to explore root endosymbioses in the model legume Medicago truncatula. Journal of Biotechnology 2004, 108:95-II 3 .

44. Able AJ: Role of reactive oxygen species in the response of barley to necrotrophic pathogens. Protoplasma 2003, $221(1): 137-143$.

45. Kulikova O, Gualtieri G, Geurts R, Kim D, Cook D, Huguet T, de Jong JH, Fransz PF, Bisseling T: Integration of the FISH pachytene and genetic maps of Medicago truncatula. Plant Journal 200I, 27(I):49-58.

46. Young ND: QTL mapping and quantitative disease resistance in plants. Annual Review of Phytopathology 1996, 34:479-50I.

47. Luo ZW, Potokina E, Druka A, Wise R, Waugh R, Kearsey MJ: SFP genotyping from affymetrix arrays is robust but largely detects cis-acting expression regulators. Genetics 2007 176(2):789-800.

48. Zhu H, Cannon SB, Young ND, Cook DR: Phylogeny and genomic organization of the TIR and non-TIR NBS-LRR resistance gene family in Medicago truncatula. Mol Plant Microbe Interact 2002, I 5(6):529-359.

49. Friesen TL, Meinhardt SW, Faris JD: The Stagonospora nodorumwheat pathosystem involves multiple proteinaceous hostselective toxins and corresponding host sensitivity genes that interact in an inverse gene-for-gene manner. Plant Journal 2007, 5 I (4):68I-692.

50. Salter RM, Leath KL: Spring back stem and leafspot resistance. In Standard Tests to Charaterise Alfafa Cultivars 3rd edition. Edited by: Fox CC, Berberet R, Gray FA, Grau CR, Jessen DL, Petersen MA North American Alfafa Improvement Conference, Beltsville, MD ; 1992.

51. Duckett JG, Read DJ: The use of fluorescent dye 3,3'-dihexyloxacarbocyanine iodide, for selective staining of ascomycetes fungi associated with liverwort rhizoids and ericoid mycorrhizal roots. New Phytologist 1991, I I 8:259-272.

52. Medicago truncatula sequencing resources [http://www.med icago.org/genome/]

53. MultiQTL - the best QTL mapping software [http://www.mul tiqtl.com/]

54. Lichtenzveig J, Bonfil D J., Zhang HB, Shtienberg D, Abbo S: Mapping quantitative trait loci in chickpea associated with time to flowering and resistance to Didymella rabiei the causal agent of Ascochyta blight. Theor Appl Genet 2006, I I 3(7): I357-1369.
Publish with Biomed Central and every scientist can read your work free of charge

"BioMed Central will be the most significant development for disseminating the results of biomedical research in our lifetime. "

Sir Paul Nurse, Cancer Research UK

Your research papers will be:

- available free of charge to the entire biomedical community

- peer reviewed and published immediately upon acceptance

- cited in PubMed and archived on PubMed Central

- yours - you keep the copyright
BioMedcentral 